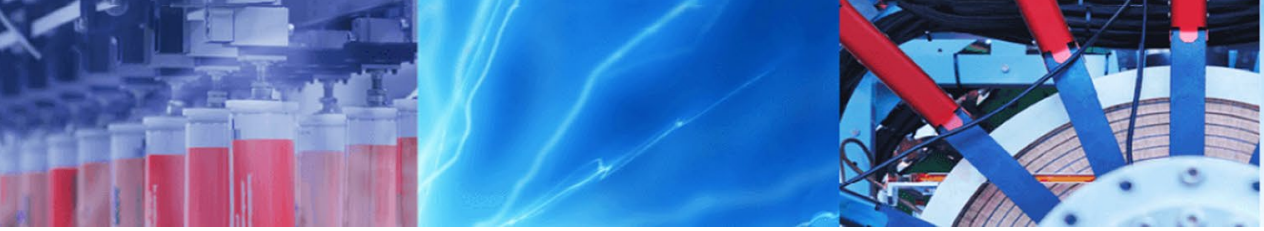

Research Article

\title{
Progressive collapse risk and reliability of buildings encountering limited gas-pipe explosion after moderate earthquakes
}

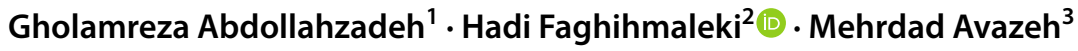

Received: 10 September 2019 / Accepted: 12 March 2020 / Published online: 16 March 2020

(c) Springer Nature Switzerland AG 2020

\begin{abstract}
Nowadays, the common urban buildings are designed so that after moderate earthquakes that may strike them many times during their lifespan, no structural damage occurs and the buildings remain in operational state. One of the risks always endangering the safety and operation of the buildings after these earthquakes is the confined explosions such as the explosion of gas pipes located in the buildings. Despite its importance, less attention is paid to this thread in design of buildings. In this study, by presenting a probabilistic approach, the relationship to determine the risk of collapse and reliability of building is presented. As a case study, effect of the moderate earthquake on a four-story steel building with special moment frames and probability of sequence explosion of gas pipes in probable locations in ground floor have been investigated. Then, the resistance capacity of the damaged building under moderate earthquake followed by explosion against the progressive collapse has been evaluated. Finally, the probability of progressive collapse and reliability of the structure were calculated. Also the explosive weight ranges for building, for different scenarios, such as safety, minor damage is determined and finally, building's collapse risk and reliability have been determined by a probabilistic method and the results have been explained.
\end{abstract}

Keywords Moderate earthquake · Confined explosion · Progressive collapse · Nonlinear dynamic analysis · Risk assessment

\section{Introduction}

Damage sustained by buildings in the past earthquakes indicates the necessity of logical methods to predict the collapse probability and probable damage of existing buildings in future earthquakes. The philosophy of designing buildings against earthquake in the current codes is prohibition of structural damage and minimization of nonstructural damage under moderate ground motion happening many times during their lifespan. Despite regarding the provisions in seismic design of buildings, the incidence of such damage and the lack of capability of exploiting them are probable. Among the most important threats the earthquake may cause for buildings is the limited explosion in the gas system of the engine plant of the building after the earthquake due to mechanical damage of the gas piping system and its connections. The finite blasts are the explosions that happen in closed spaces like tanks, procedural installations, pipes, closed rooms and underground equipment. One of the factors causing damage of gas pipes is lack of providing flexibility for them. From the other view, corrosion, leakage, unsuitable welding and collision of the pipes are among the factors causing damage. The explosion of gas-pipeline has occurred in past earthquakes but less attention is paid to it. In this study, the risk of the progressive collapse of buildings under moderate earthquakes followed by gas-pipe explosions is evaluated.

Hadi Faghihmaleki, h.faghihmaleki@gmail.com | ${ }^{1}$ Faculty of Civil Engineering, Babol University of Technology, Babol, Iran. ${ }^{2}$ Department of Civil Engineering, Ayandegan Institute of Higher Education, Tonekabon, Iran. ${ }^{3}$ Faculty of Civil Engineering, Amol University, Amol, Iran. 
When one or more structural members of the building are destroyed suddenly due to the explosion, each load distribution may cause fracture of other structural elements one after another and the building may collapse progressively. Among the first researchers studying the progressive collapse are [19] who presented various approaches to mitigate this issue. The current procedure for designing the structure against the explosion is designing against local failure of elements in first step and then using tactful measures to prevent spread of damage to other elements in next step. Nowadays, evaluation techniques and risk management procedures for decreasing vulnerability derived from natural disasters and man-made hazards have been paid attention a lot. Stewart et al. [28] have studied evaluation of the possible dangers arisen from explosion terror-stricken damage to infrastructures. In this review, the problems about the risk assessment including the risk transfer concept as well as comparisons with natural hazards have been discussed. Asprone et al. [5] have presented a probabilistic model to evaluate the hazardous risk along with limit state of collapse for a reinforced concrete building exposed to explosive threats located in a seismic site. Parisi and Augenti [23] assessed the performance of seismic resistant RC frame structures designed according to Eurocode 8 code, under blast loadings. They developed the intended blast scenarios in accordance with structural site quality and the amount of explosives required. Then, to evaluate the robustness of the building, they performed a pushdown analysis. Fu [13] advanced a new method to estimate a tall building's response to blast loadings. He finally offered a comparison between his proposed method and alternative path method (APM). In this study, by presenting a probabilistic approach, the relationship to determine the risk of collapse and reliability of building is presented. And as a case study, effect of the moderate earthquake on a four-story steel building with special moment frames and probability of sequence explosion of gas pipes in probable locations in ground floor have been investigated. On the other side, one can observe that in most moderate earthquakes along with critical earthquake load, gas explosive can increase the damages subjected to a structure. This factor highlights the significance of performing the current study so that one can evaluate the collapse rate of the structure with an appropriate method under such condition and also obtain an acceptable and correct assessment of structures subjected to these two critical loads.

\section{Definition of the explosion phenomenon}

Explosion is the sudden release of energy that can be as gaseous combustion, nuclear explosion or variety of bombs. The TNT unit is usually used as a reference to determine the explosion power. When an explosion wave hits a building, the building is under the load derived from the tension and pressure forces of the explosion wave. Among the main characteristics of an explosion which impose force on the structure, the random explosion situation, dynamic and transitory and the short time of action (between few milliseconds to few seconds) can be pointed. Each blast load is determined by three parameters: the wave shape, the peak overpressure $\left(P_{s o}\right)$ and time duration $\left(t_{d}\right)$. Based on the explosion origin, blast waves are divided into two shapes: the impact wave and the pressure wave. The impact wave is resulted from explosion of solid explosives in which the gas pressure formed by explosive propagates from explosion source and increases to the base pressure $\left(P_{s o}\right)$, then decreases to the environmental pressure. This stage is referred as positive phase. As a result of the wave propagation, the gases derived from explosion are cooled and their pressure is reached to a negligible amount lower than the atmosphere pressure. Due to this pressure difference, the current direction gets reversed towards the explosion center. The result of this action is decrease in the pressure or stuck that is called negative phase $[1,15]$. The pressure of the negative phase is relatively small and gradual so it is conservatively ignored in designing the resistant buildings against explosion (Fig. 1).

Pressure wave arises from gas or liquid explosive and as shown in Fig. 2, first, increases up to overpressure $P_{\text {so }}$ and then decrease to environmental pressure $P_{o}$ without negative phase. Where $t_{d}$ is called explosion duration.

\section{Measurement of blast power}

The most common method for determining the explosion power is the Hopkinson-Cranz measure or the cube rule. This rule was described by Hopkinson [16] for first time and developed by Cranz [9]. This rule indicates that two similar explosive charges exploded with similar geometries but with different sizes, in the similar atmospheric conditions; create similar explosive waves when they

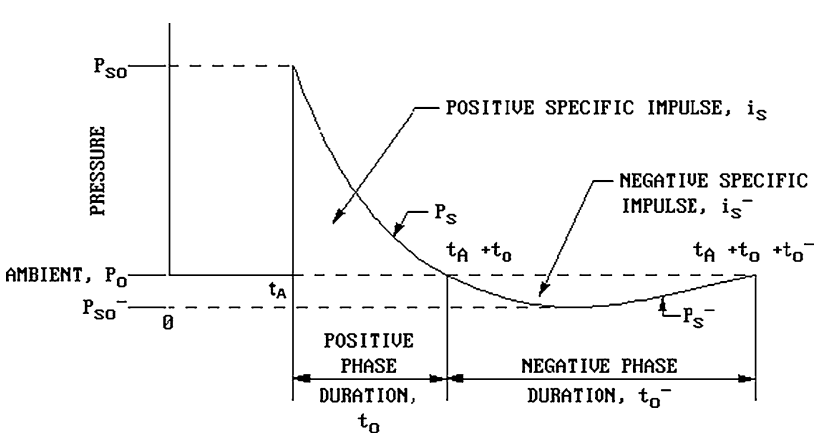

Fig. 1 Impact wave [29] 


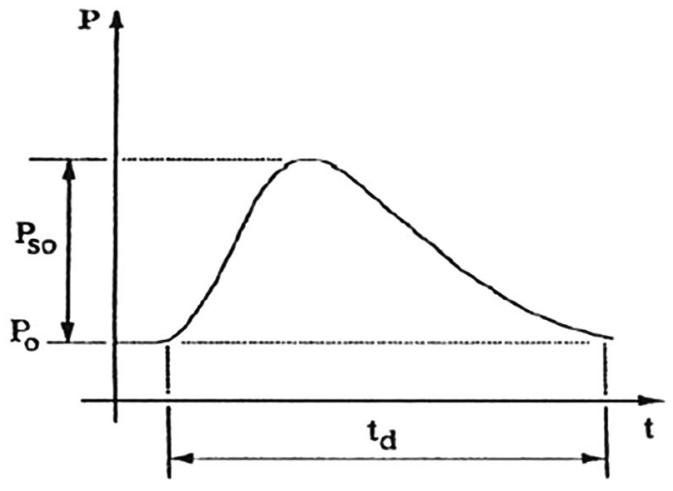

Fig. 2 Pressure wave [29]

have similar scaled distances [26]. The scaled distance is a dimensional parameter determined by the Eq. (1),

$z=\frac{R}{w^{1 / 3}}$

where $\mathrm{Z}$ is the scaled distance; $\mathrm{R}$ is the standoff distance $(\mathrm{m})$ and $\mathrm{W}$ is the explosives weight of equivalent TNT in kilogram. The Hopkinson-Cranz equation is only valid for explosion resulted from TNT and the TNT unit is used as a reference to determine the explosion power.

For other explosives, the equivalent TNT weight should be determined. In most cases, the natural gas is used that for which the equivalent TNT weight is obtained by Eqs. (2) and (3) [18]:

$E=\alpha \cdot \Delta H_{C} \cdot m_{F}$

$W_{T N T}=\frac{E}{4500}$

where $\mathrm{E}$ is the explosive energy released in $\mathrm{KJ}, \alpha$ is yield coefficient, the fraction of combustion energy participating in blast wave, which is typically in the range of $1 \%(0.01)$ for unconfined mass releases to $100 \%$ (1.0) for confined vapor releases, $\Delta H_{c}$ is the theoretical net heat of combustion in $\mathrm{KJ} / \mathrm{Kg}, m_{F}$ is the mass of flammable vapor release in $\mathrm{kg}, \mathrm{W}_{\mathrm{TNT}}$ is the equivalent TNT weight in $\mathrm{Kg}$. Heat of combustion $\left(\Delta H_{c}\right)$ for some combustible gases is shown in Table 1.

\section{Blast loading}

The calculation of blast loads has been performed by UFC code, edition 4-023-03 [31], which is the most valid and complete reference for blast loading. Generally, in considering the effect of explosion on target, the following factors are focused:
Table 1 Combustion heat for some flammable gases [18]

\begin{tabular}{lc}
\hline Flammable gas & $\Delta H_{c}(\mathrm{~kJ} / \mathrm{kg})$ \\
\hline Acetylene & 482,220 \\
Ethane & 47,490 \\
Methane & 50,030 \\
Propane & 46,360 \\
Hydrogen & 130,800 \\
\hline
\end{tabular}

- The weight of the explosive

- The distance of the explosion center to the target

- The geometry of the building's structural system

- The reflection angle of blast wave

When the building is hit by blast wave, it is loaded by overpressure and suction forces due to blast. The suction forces are ignored because they are relatively small and gradual. In first step, according to scaled distance (z), the required parameters for blast loading is determined. The surface of the building against the explosion source tolerates a reflected pressure $\left(p_{r}\right)$ that is dependent on the angle of the blast wave with the target reflection surface. It is assumed that walls resist blast wave and conservatively, the openings are ignored. For inside explosion, in addition to reflection pressure $\left(p_{r}\right)$, gas pressure $\left(p_{g}\right)$ also develops which is dependent on the openings area. The gas pressure is far less than the reflection pressure and its duration is far more than the reflection pressure inside the building as shown in Fig. 3.

\section{Dynamic characteristics of the materials exposed to blast}

In order to model a structure, well knowing of the structure is necessary. One of the most significant realizations of a structure model is material behaviors. Blast loads typically produced very high strain rates in the range of $10^{2}-10^{4} \mathrm{~s}^{-1}$. This high straining rate would alter the dynamic mechanical properties of target structures and accordingly, the expected damaged mechanisms for various structural

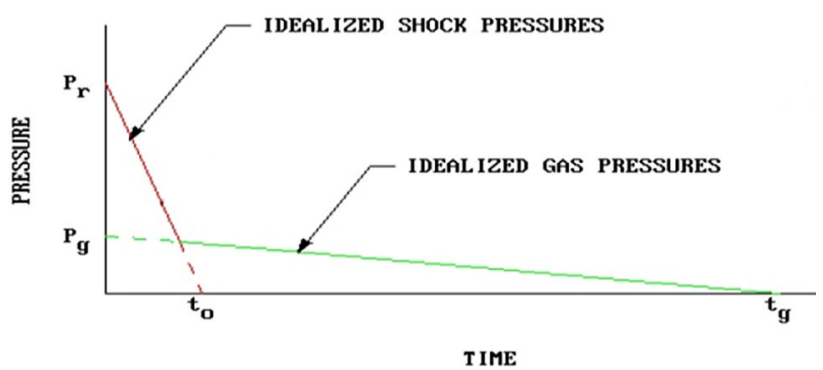

Fig. 3 The ideal curve of the explosion charge [26] 
elements. For steel structures subjected to blast effects, the strength of steel can increase significantly due to strain rate effects [2]. Figure 4 shows the approximate range of the expected rates for different loading conditions [21].

The response of the materials under the blast loads is noticeably different from that under the static loads. In the former, due to the rapid loading, materials' resistance increases significantly compared to the latter. In the rapid loading, the materials cannot deform with the similar rate as imposed load. This feature causes increment in both yield stress and ultimate stress before failure as shown in Fig. 5.

To define the steel behavior under the blast load, real stress-strain curve of steel (Type of St37) should be used. Since it is possible that the steel column enters the plastic area under the blast loading, the steel behavior in the plastic area must be defined. Also plastic strain is the strain in the area of plastic minus the elastic strain related to the surrender strain. The sensitivity of the strain rate is expressed by Eq. (4) [6] where $\sigma_{y}$ is the yield stress considering the effect of the strain rate, $\dot{\varepsilon}^{p l}$ is the rate of the plastic stain, $\gamma$ is the viscosity parameter, $m$ is the strain hardening parameter and $\sigma_{y}$ is the static yielding stress. When $\dot{\varepsilon}^{p l}$ approaches zero in low rate loading, or when $\gamma$ goes to infinity, the solution approaches the static solution (independent of the rate). The values suggested for the structural steel are $\gamma=40 \mathrm{~s}^{-1}$ and $m=0.2$.

$\sigma_{y}^{\prime}=\left[1+\left(\frac{\dot{\varepsilon}^{p l}}{\gamma}\right)\right]^{m} \sigma_{y}$

To account for the increase in the strength of the materials due to rapid strain-rate, the static strength of the steel is multiplied by dynamic amplification factors given in Table 2.

\section{Progressive collapse}

The progressive collapse is the spread of the local failure from one member to another that results in the collapse of the whole structure or its major part. Generally, buildings are not designed for special loading conditions such as the blast. But even when they are designed for wind or earthquake, no proper preparations are considered for the local damage or

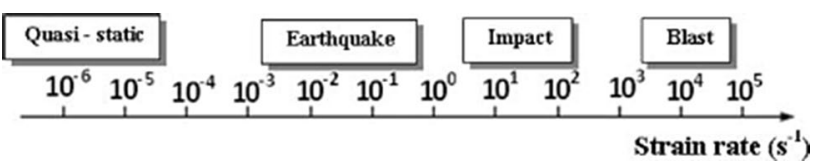

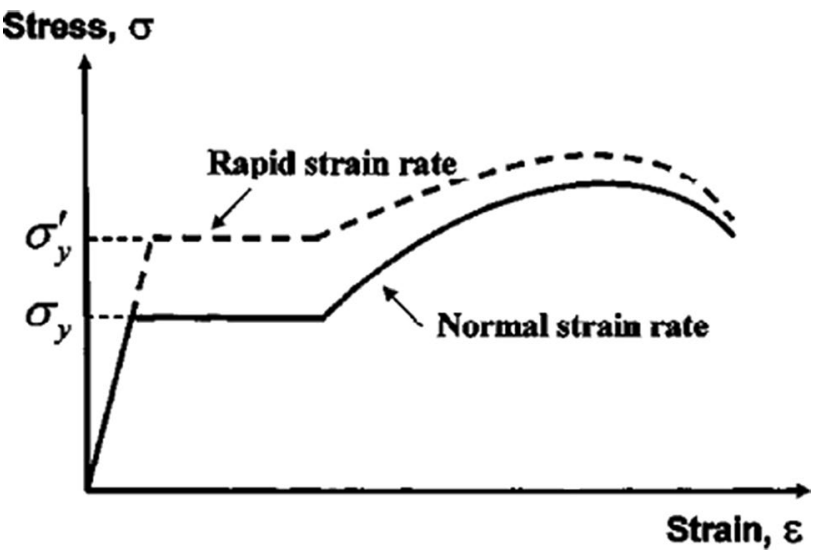

Fig. 5 Stress-strain curve of steel considering the effect of the strain rate [6]

failure modes. Hence, they may be damaged seriously under such loadings.

To reduce the possibility of progressive collapse in buildings, two general patterns have been proposed [29]:

(a) The direct design which includes two approaches: special local strength and alternative path.

(b) The indirect design which includes suggestions for decreasing the progressive collapse of structures such as the suitable plan-design and the allied mortising in the structure (Tie forces) (UFC [31]. In this method, an alternative path method was used which is briefly described below.

The main idea of alternative path method is that the structure is designed so that there are other alternative pathways to carry the load to the ground if the routine load-carrying pathways are eliminated or damaged. Assessing alternative paths is performed by three methods, linear static, nonlinear static and nonlinear dynamic analyses. The nonlinear dynamic analysis considers both effects of the material nonlinearity and geometric nonlinearity and dynamic effects, so, it shows the most elaborated results but it is complicated and time-consuming. Furthermore, two-dimensional models are not allowed in this method [31].

It should be noted that, the nonlinear dynamic analysis in the alternative path method is performed according to UFC 4-023-03 [31]. In this way, the gravity load combination of $1.2 \mathrm{DL}+0.5 \mathrm{LL}$ and the lateral load of $0.002 \Sigma \mathrm{P}$ are applied to the structure simultaneously as

Table 2 Dynamic amplification factors to account for rapid strain rate [29]

\begin{tabular}{lll}
\hline Component & $\sigma_{y}$ & $\sigma_{u}$ \\
\hline Beam & 1.29 & 1.1 \\
Column & 1.1 & 1.05 \\
\hline
\end{tabular}

Fig. 4 Strain rates associated with different types of loading [21] 


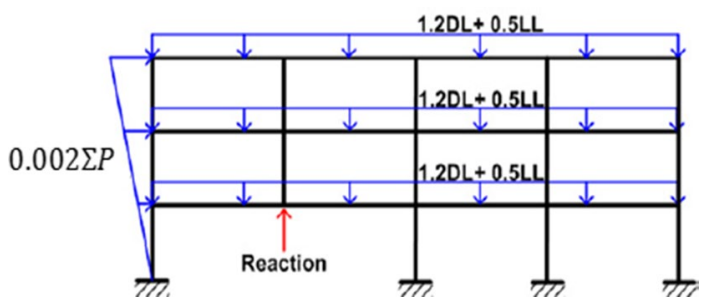

Fig. 6 Applied loads in nonlinear dynamic analysis

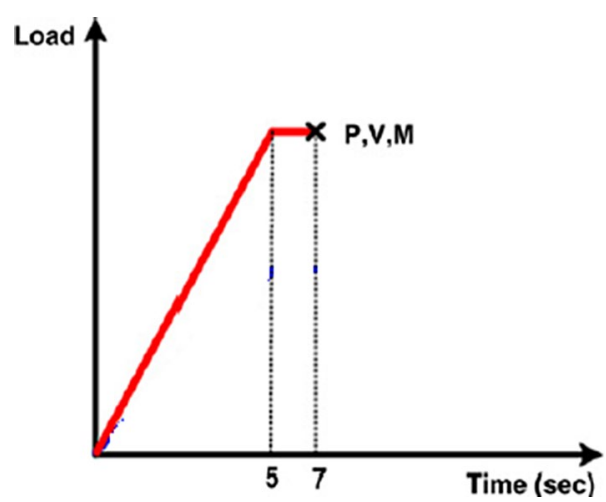

Fig. 7 Simulation of the dynamic effects due to sudden omission of the column

shown in Fig. 6. The lateral load is applied in four directions. $\mathrm{DL}$ is the dead load; $\mathrm{LL}$ is the live load and $\Sigma \mathrm{P}$ is sum of dead and live loads. For each blast scenario, which is accompanied by omitting the defective columns, four analyses have been performed and the most critical one has been considered.

To simulate the dynamic effects after sudden omitting of the column, at first, axial force (P), shear force (V) and bending moment $(\mathrm{M})$ of the column under the gravity load are substituted instead of the omitted column. As shown in Fig. 7, this alternative force increases for $5 \mathrm{~s}$ to reach its maximum, and then it remains unchanged for $2 \mathrm{~s}$ until the system reaches the equilibrium state. Finally in seventh second, it is removed suddenly in order to dynamic effects of sudden omission of the column are taken into account.

The acceptance criteria for beams and columns are collapse prevention (CP) and life safety (LS) respectively. The definition of these target performance levels are presented in Table 3 for both structural and nonstructural components. Figure 8 , is shown target building performance levels and ranges. In fact, if the plastic hinge formed in beams or columns don't qualify for these acceptance criteria, the structure loses its usability and the collapse happens.

\section{Probable locations of gas explosion and determination of equivalent weight of TNT}

Today natural gas is often used in heating system of buildings. After earthquake that hurts gas piping systems and their installation, explosion occurs in power house that usually resides at the first floor of the building. To assess the effect of gas explosion, first, probable locations of explosion must be determined. These points should be considered in a way that can simulate explosive event scenario realistically as much as possible.

By considering plan of considered building, location of power house in ground floor is investigated and probability of each location of power house is determined. Then for any possible situation, a number of possible points for the explosion is considered and $x$ and $y$ distances between the points to building's columns is determined. The number and coordinates of these points is determined that all critical points of a possible gas explosion accident are covered.

The most common composition of natural gas is methane $\left(\mathrm{CH}_{4}\right)$. Also, there are heavier hydrocarbons like Ethan, propane, Bhutan and etc. and impurities like sulfur with different amounts. Chemical composition of natural gas in Iran is presented in Table 4 [24].

As shown in Table 4, most part of natural gas is consisted of methane. So, for determining equivalent weight of TNT of the leaking gas through the pipe injured by earthquake, corresponding value for methane in Table 1 is applied. Unexpected high volume leakages caused by broken piping systems, in a short time, fill the environment and make it ready for explosion. The amount of gas leaking through the broken pipe depends on gas pressure and hole diameter. Spreading rate of Methane from sources with different hole dimension and pressure is shown in Fig. 9.

Diameter of hole in damaged pipe and leaking gas weight are determined in order that the building experiences the least and the most failures realistically.

\section{Simulation of the blast load}

Simulation of the blast effect is done by ABAQUS software. Regarding the fact that the columns experience significant damage under blast loads and the importance of them in load-carrying capability, stability and progressive collapse of the building, it's assumed that the blast load is a decreasing triangular impact versus time, uniformly applied only to the columns of each 

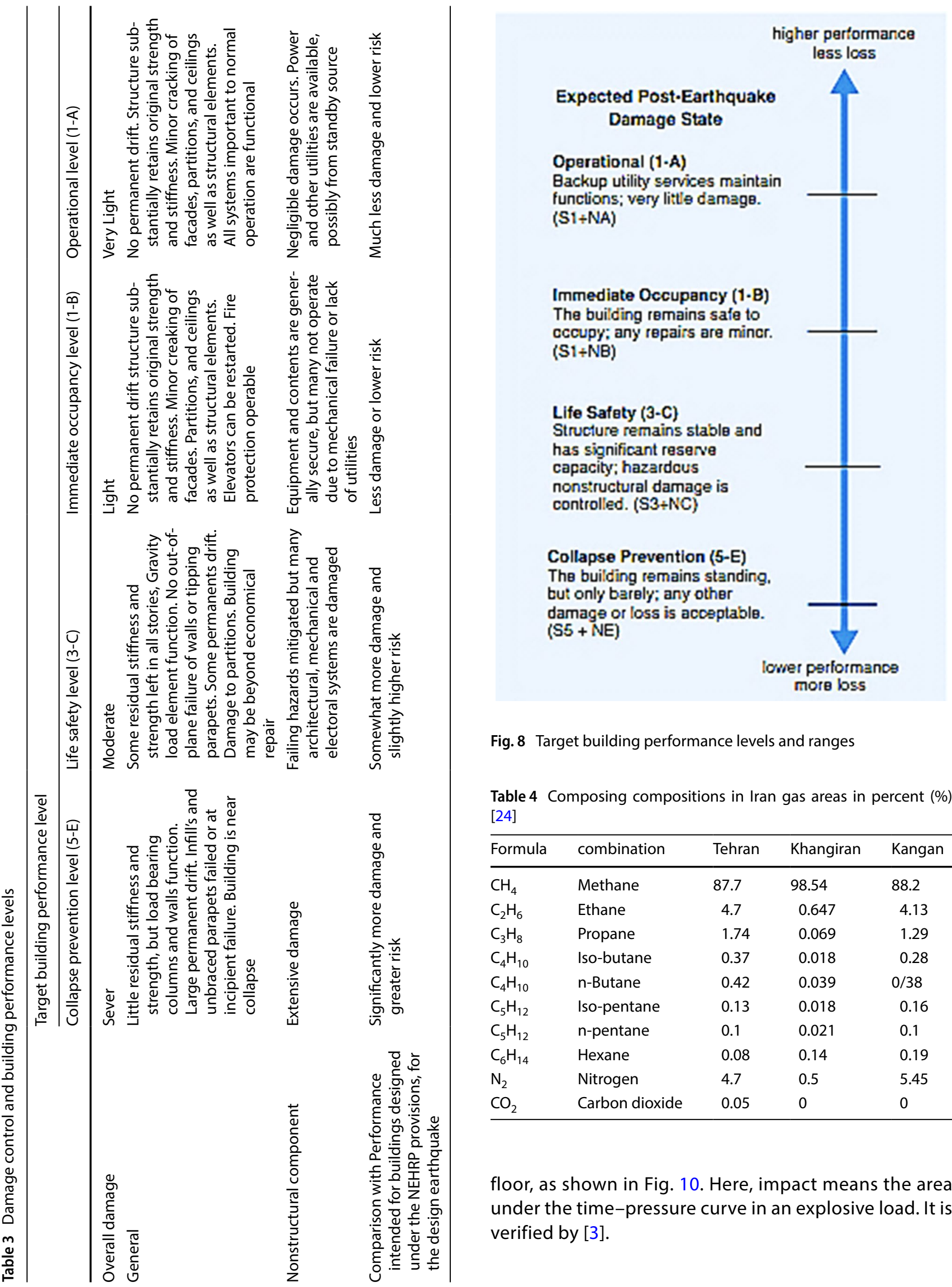

Fig. 8 Target building performance levels and ranges

Table 4 Composing compositions in Iran gas areas in percent (\%) [24]

\begin{tabular}{llclc}
\hline Formula & combination & Tehran & Khangiran & Kangan \\
\hline $\mathrm{CH}_{4}$ & Methane & 87.7 & 98.54 & 88.2 \\
$\mathrm{C}_{2} \mathrm{H}_{6}$ & Ethane & 4.7 & 0.647 & 4.13 \\
$\mathrm{C}_{3} \mathrm{H}_{8}$ & Propane & 1.74 & 0.069 & 1.29 \\
$\mathrm{C}_{4} \mathrm{H}_{10}$ & Iso-butane & 0.37 & 0.018 & 0.28 \\
$\mathrm{C}_{4} \mathrm{H}_{10}$ & n-Butane & 0.42 & 0.039 & $0 / 38$ \\
$\mathrm{C}_{5} \mathrm{H}_{12}$ & Iso-pentane & 0.13 & 0.018 & 0.16 \\
$\mathrm{C}_{5} \mathrm{H}_{12}$ & n-pentane & 0.1 & 0.021 & 0.1 \\
$\mathrm{C}_{6} \mathrm{H}_{14}$ & Hexane & 0.08 & 0.14 & 0.19 \\
$\mathrm{~N}_{2}$ & Nitrogen & 4.7 & 0.5 & 5.45 \\
$\mathrm{CO}_{2}$ & Carbon dioxide & 0.05 & 0 & 0 \\
\hline
\end{tabular}

floor, as shown in Fig. 10. Here, impact means the area under the time-pressure curve in an explosive load. It is verified by [3]. 


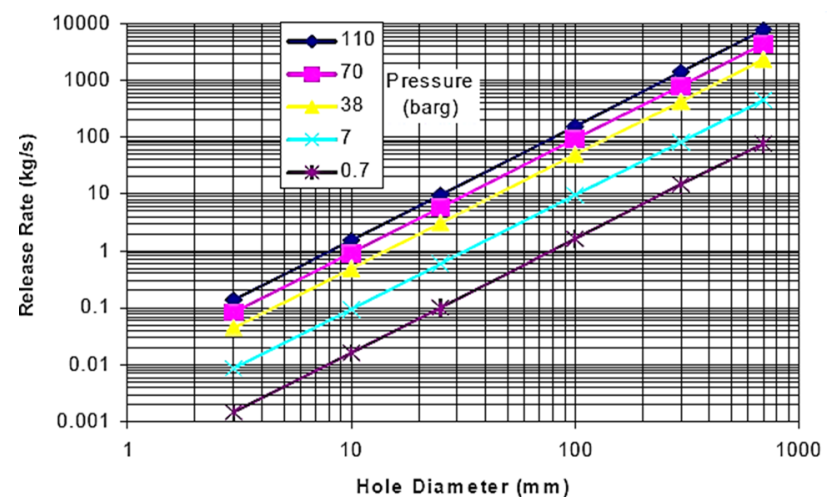

Fig. 9 Methane's spreading rate from dropping source [7]

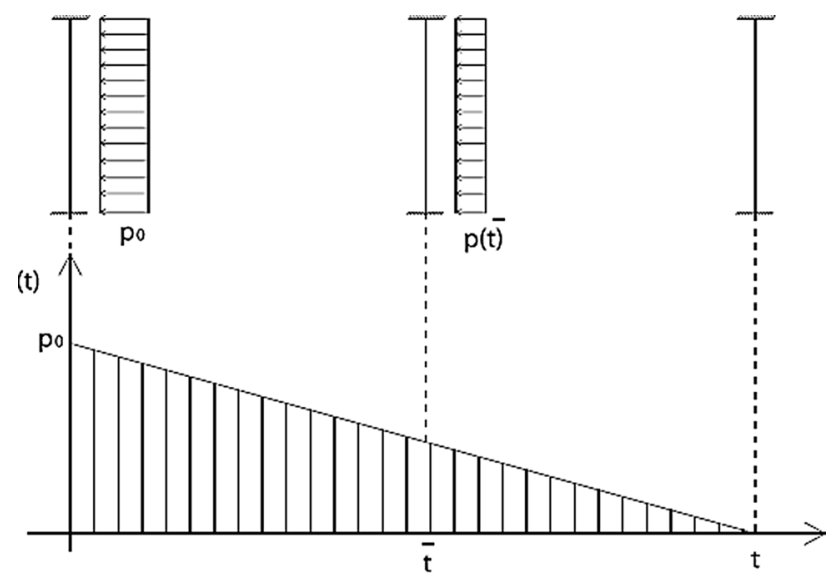

Fig. 10 The time-pressure curve of the blast load on the columns $[3,5]$

The columns are modeled by the S4R shell element which is four-node shell elements with reduced integration to prevent shear locking. In S4R element, the nonlinear effects resulted from the extra shear under the blast load are considered more carefully in comparison to the other elements and the element has high convergence rate in the nonlinear analyses. As shown in Fig. 11, this element has several manners in hourglass form that can extend all over the mesh.

\section{Effects of axial load on the column under the blast load}

The amount of the axial load in the columns exposed to explosion is dependent on the geometry, support conditions and the slenderness. Although according to the researches, including the axial load in analyzing of the column under explosion with the slenderness coefficient
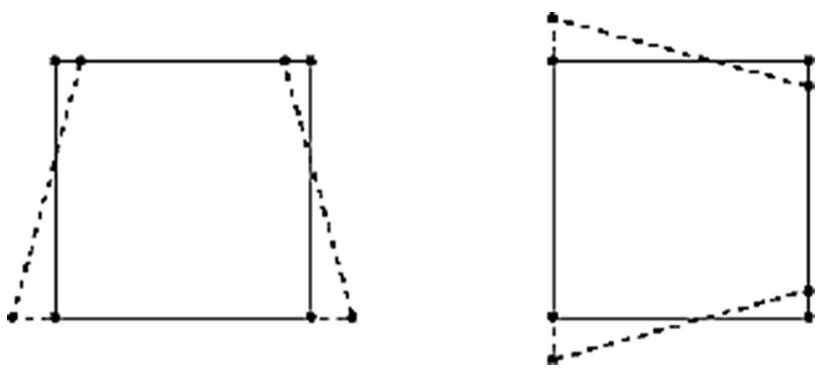

Fig. 11 Shell element S4R [22]<smiles>[Y][Y]</smiles>

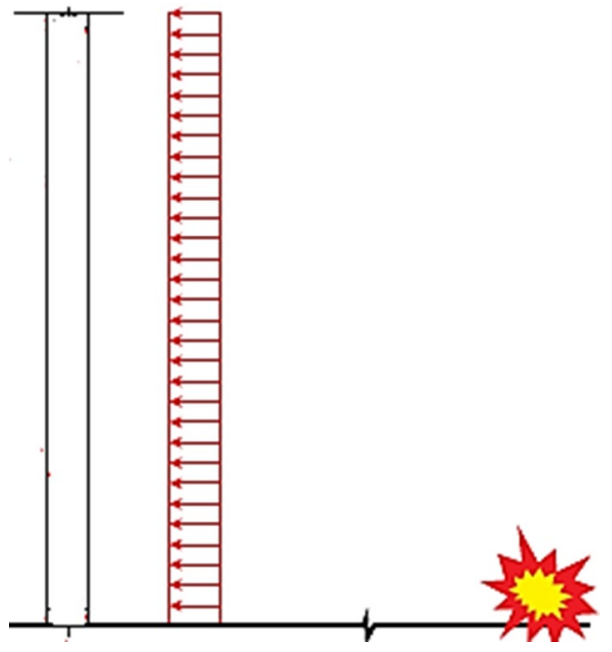

Fig. 12 The axial load effect on the column exposed to the explosive load [11]

lower than 38 can be ignored [11, 12], but for more confidence in this research it has been considered (Fig. 12).

\section{Analysis of the column exposed to blast}

For dynamic analysis of the columns under the blast load, the Dynamic Explicit Analysis has been used. In high-rate dynamic phenomena like the explosion in which dynamic analysis of the model in a short time span is aimed and also in cases that the material may experience fracture, crack or failure, this analysis method is recommended in ABAQUS. The velocity of the nodes and elements are continuously changing in this analytical method. So, the results obtained from this procedure are accurate and stable provided that the analysis time step be small enough to capture variability of velocity and acceleration of the 
nodes and elements. The geometrical nonlinear effects are taken into account with activating the N1geom option. During the analysis, response of the columns is recorded in $0.05 \mathrm{~s}$.

\section{Failure criterion}

In order to determine failure state of the columns due to the blast, the Von Mises yield criterion is applied. The yield criterion determines the boundary between the elastic and plastic behavior and shows the stress level in which the plastic deformation initiates. The Von Mises yield criterion is the most common criterion used for metals. According to this criterion, yielding of a section of the column occurs when the Von Mises stress reaches the yield stress. Here, failure does not necessarily mean fracture. The deformation resulted from the material yielding causes the member to lose the tolerability of the load and to get unusable. For instance, for the column shown in Fig. 13, the Von Mises stress has exceeded the yield stress ( 330 $\mathrm{Mpa}$, by taking into account the effect of the strain rate) under the blast load and design axial force in the moment of $0.0025 \mathrm{~s}$ and the column has lost its load-carrying ability and has become unusable.

\section{Removing the column in the alternative path method}

While removing the column from the building, the continuity of the beams in both sides of the column should be maintained. It means that the panel zone should not be damaged and only the column must be removed from the building. Empirically, removing the panel zone causes loss of continuity of the adjacent beams and no chance remains for the structure [31]. Figure 14 shows the right and wrong methods for removing the column. Which indicate that for each column that cannot provide the required vertical tie force; remove the clear height between lateral restrains [31].

\section{Progressive collapse}

To assess the progressive collapse for the columns, after analyzing the model happening under probable explosive-terroristic scenarios inside and outside of the building, a dynamic nonlinear analysis is followed according to the UFC [31]. To evaluate the members experiencing inelastic deformation, characteristics of plastic hinges should be determined according to the FEMA-356 [10]. In this way, the $\mathrm{M} 3$ moment hinge is assigned to the both end-points and mid-span of the beams and the P-M2-M3 interaction hinge is assigned to the both end-points of the columns. The progressive collapse analysis is carried out under the combination of $1.2 \mathrm{DL}+0.5 \mathrm{LL}$ gravity loads as well as the $0.002 \Sigma \mathrm{P}$ lateral loads. Exceeding CP performance point in plastic hinges of the beams and LS performance point in those of the columns indicate the incidence of the progressive collapse in the building. Figure 15 shows a case of the progressive collapse occurrence.

\section{Risk assessment and reliability}

Risk assessment is a logical method to determine, qualitatively and quantitatively, the amount of the dangers and potential outcomes derived from the possible events on individuals, materials, equipment and environment.
Fig. 13 Von Misses strain state in the steel column in the maximum level of the stress
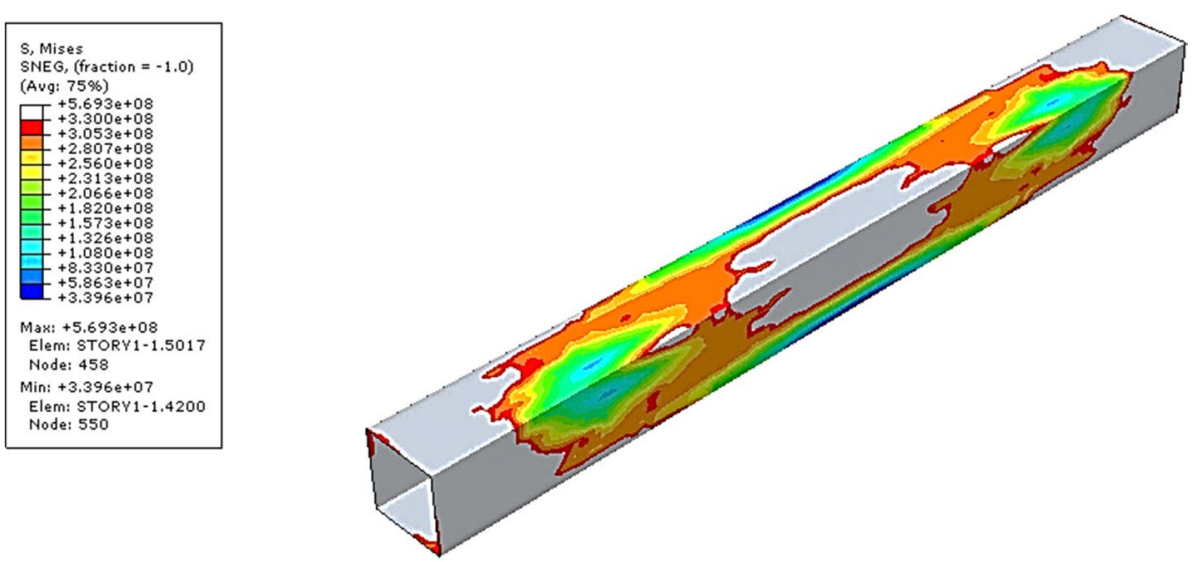

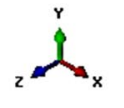

Step: Step -

Increment 1218: Step Time $=2.5012 \mathrm{E}-03$

Primary Var: S, Mises

SN Applied Sciences 


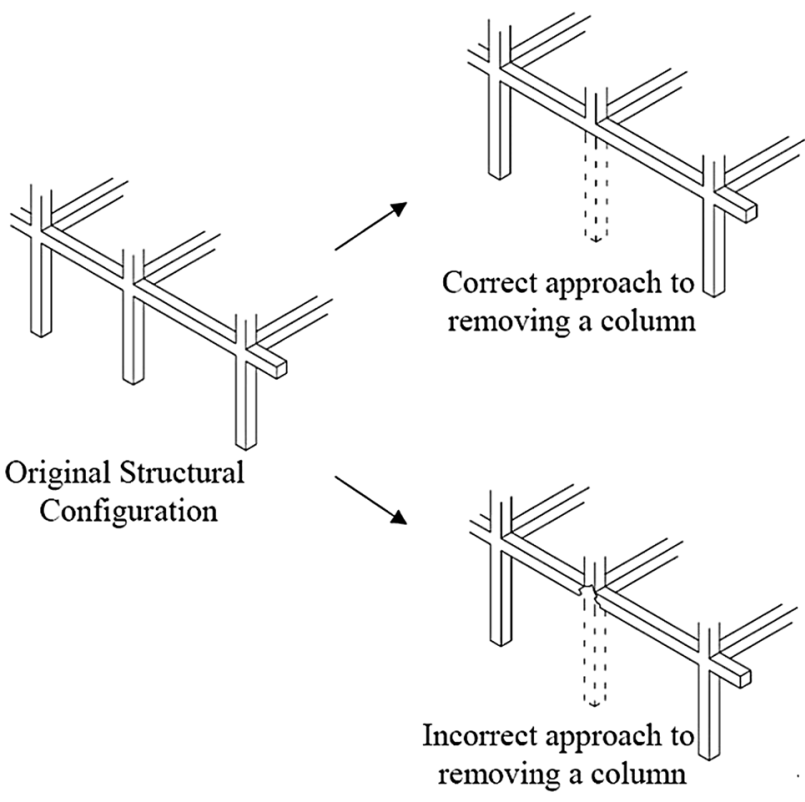

Fig. 14 Removing the column from the building in two manners, true and false [31]

Complex uncertainties of critical events probable in lifetime of strategic structures, makes the probabilistic assessment of the structural performance inevitable. Collapse probability of the structure under critical events during its lifespan can be written in conditional probability form as Eq. (5) $[8,14,20]$ :

$P(C)=\Sigma P(C / A) P(A)$

where A stands for a critical event such as explosion, earthquake, storm, etc., $P(C)$ is the collapse probability, $P(C / A)$ is the collapse probability conditioned on the critical event $A$ and $P(A)$ is the occurrence probability of critical event $A$.

One of critical events endangering immunity and operation of common today buildings after medium earthquakes is limited explosion in their gas pipeline and gas power house. Considering the earthquake followed by gas-pipe explosion as critical event, the collapse probability can be written as:

$P(C)=\Sigma P(C \mid E Q \& B$ last $) P(E Q) P($ Blast $)$

where $\mathrm{P}(\mathrm{C} \mid \mathrm{EQ} \& \mathrm{Blast})$ is the collapse probability conditioned on earthquake event followed by gas explosion, $P(E Q)$ is earthquake event probability and $P(B l a s t)$ is explosion event probability.

The concept of the reliability has been interpreted in several different ways and with different methods; the most common definition of the reliability introduces it as the probability of that a sample performs a given task in a given period and under specified operating conditions.

Because of uncertainties, the reliability should be considered in a probabilistic framework. In analysis and design of the building, the reliability has been defined as the probability of that the structure does not exceed any of determined limits during its life pan. The reliability $\left(R_{0}\right)$ is defined in terms of the collapse probability, $\mathrm{P}(\mathrm{C})$, as following [31]:

$R_{0}=1-P(C)$

\section{Numerical example}

A possible application of the methodology described in the previous section can refer to calculation of collapse probability and building reliability of a generic four story steel framed building. A numerical example is here presented; the characteristic of the case study structure are outlined in the following.

\subsection{Structural model description}

The indeterminate structural systems like moment frames has appropriate ability and capability for the redistribution of extra loads among the remaining structural elements while other structural systems with lower degrees
Fig. 15 The occurrence of failure in progressive collapse analysis
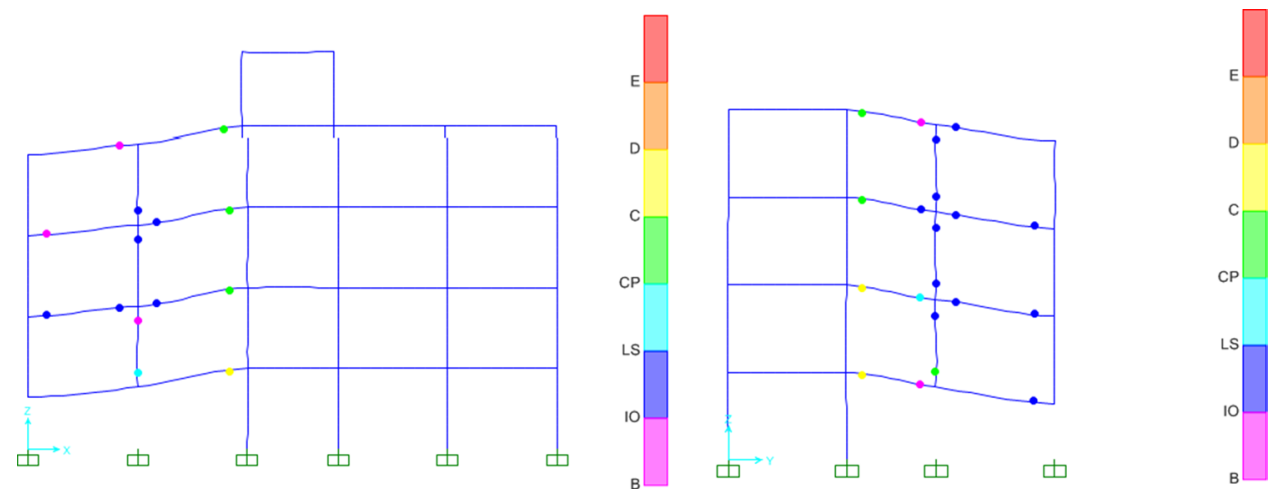

SN Applied Sciences A SPRINGER NATURE journal 
of indeterminacy like simple frames experience infinite deformations under blast loads and don't have capability of redistribution of extra loads. Structural steels in classes St37 and St52 have sufficient ductility for designing against blast. In most of the time, it should be avoided to use materials with high strength for preventing problems due to decreasing the ductility. The st37steel is proper and common for usual and blast loads applied on structures [26]. According to these comments, the studied model is a four story steel structure with special moment resisting frame system in both $X$ and $Y$ direction, with great importance, and is located in very high seismic zone. Columns to foundation connections are rigid. Floor slabs are oneway joist block and pure height of each story is $2.8 \mathrm{~m}$. The limit state or LRFD method is used to design the structure against blast. AISC360-05/ LRFD [4] code is used to analyze and design in SAP 2000 [25] software. Figure 16 shows the typical plan of the stories.

Box sections are used for the columns. The section is suitable for buildings with moment resisting frames in both directions. IPE sections are also chosen for the beams. The design process is performed in several phases so that on one hand, selected sections are near the optimal sections regarding the stresses and the lateral displacements of the structure, and on the other hand, designing of particles are simple, uniform and applicable. Also, it is assumed that connections are designed for final capacity of connected member and discussion about connections was omitted. The Results are summarized in Table 5. Material parameters are outlined in Table 6 . Real stress-strain curve of St37 steel, as shown in Fig. 17, should be used.

\subsection{Selected records}

Earthquakes with magnitude 5 to 5.9 are called medium earthquakes with average 800 incidences over last 100 years (since 1900). Iranian [27] code introduces the

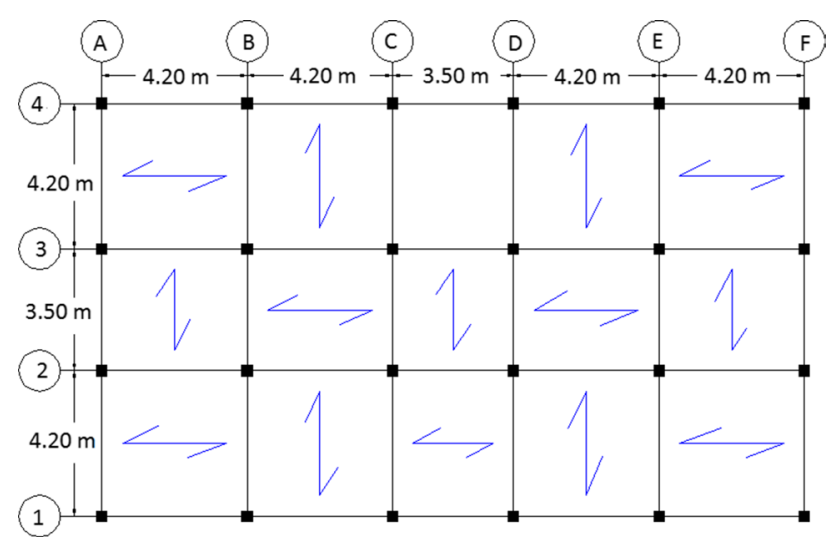

Fig. 16 Typical plan of the stories small and average earthquakes as events with exceedence probability greater than $99.5 \%$ during 50 -year design period of the building. In U.S. Army [30], average earthquakes are defined as events that with occurrence probability greater than $50 \%$ in 50-year lifespan. Peak ground acceleration of such events is taken as $0.2 \mathrm{~g}$ for some special buildings and $0.14 \mathrm{~g}$ for others. The Chinese code applies a peak ground acceleration of $0.35 \mathrm{~g}$ for moderate earthquakes in different regions [26]. Moreover, the intensity of a strong earthquake is about one degree further than that of base intensity. The records used for determination of ground motion effects must be indicator of the real movement of the site during such events. Also source-to-site distance and the seismic source mechanism should be considered in selection of records. Most earthquakes observed in Iran are shallow events, for which the damages are usually limited to the central and nearfault region. The earthquakes with the depth smaller than $15 \mathrm{~km}$ are called the shallow earthquakes.

Considering soil type II with $\mathrm{V}_{\mathrm{s}} 30$ of 375 to $750 \mathrm{~m} / \mathrm{s}$ (based on classification of reference [27] for site soil condition, four strong ground motion records with medium magnitude has been selected as listed in Table 7. It is assumed that the occurrence probability of each of these records is $25 \%$. To achieve maximum acceleration of an earthquake with moderate intensity, each of these four records are scaled to $0.2 \mathrm{~g}$ and applied to the building separately.

\subsection{Probable locations of gas explosion and determination of equivalent weight of TNT}

Regarding symmetric plan of the studied building, power house may be located in three positions shown in Fig. 18. It is assumed that the explosion occurs in each point with $33.3 \%$ probability and for each of these locations, 8 probable point of explosion are considered so that each point has a 1.5-m distance from neighboring $X$ and $Y$ axis of column points. Number and coordinate of these points are defined in order that cover all critical probable points of explosion event.

Table 5 Designed sections of members

\begin{tabular}{lll}
\hline & Beam & Column \\
\hline 1st floor & IPE270 & BOX22 $22 \times 1.25$ \\
2nd floor & IPE270 & BOX20 $20 \times 1.25$ \\
3rd floor & IPE240 & BOX18 $\times 18 \times 1$ \\
4th floor & IPE180 & BOX16 $\times 16 \times 1$ \\
& IPE160 & BOX14 $14 \times 0.8$ \\
\hline
\end{tabular}


Table 6 Material properties

\begin{tabular}{|c|c|c|c|c|c|c|c|c|}
\hline \multirow[t]{2}{*}{ Elastic properties } & \multicolumn{2}{|c|}{ Plastic properties } & \multicolumn{2}{|c|}{ Rate dependent } & \multicolumn{3}{|c|}{ Dynamic amplification factors } & \multirow{2}{*}{$\begin{array}{l}\text { General } \\
\text { property } \\
\left(\mathrm{kg} / \mathrm{m}^{3}\right)\end{array}$} \\
\hline & $\begin{array}{l}\text { Plastic strain } \\
(\mathrm{m} / \mathrm{m})\end{array}$ & $\begin{array}{l}\text { True stress } \\
(\mathrm{Mpa})\end{array}$ & Hardening & Power law & Component & $\sigma_{y}$ & $\sigma_{u}$ & \\
\hline$E=210 \times 10^{9}(\mathrm{~Pa})$ & 0 & 300 & Multiplier & 40 & Beam & 1.29 & 1.1 & $\rho=7800$ \\
\hline \multirow[t]{4}{*}{$v=0.3$} & 0.025 & 350 & Exponent & 5 & Column & 1.1 & 1.05 & \\
\hline & 0.1 & 375 & & & & & & \\
\hline & 0.2 & 394 & & & & & & \\
\hline & 0.35 & 400 & & & & & & \\
\hline
\end{tabular}

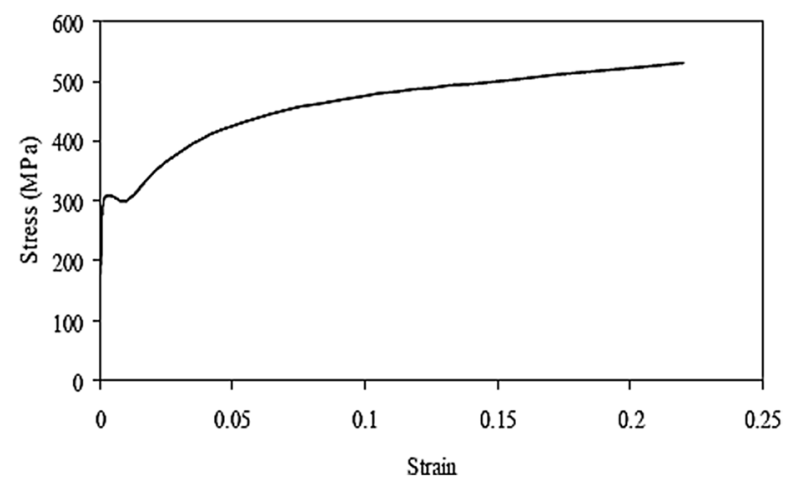

Fig. 17 True stress-strain curve of steel (ST37) [17]

Table 7 The suite of four ground motion records

\begin{tabular}{lllrl}
\hline No. & Event & Station & $R^{d}(\mathrm{~km})$ & $M^{c}$ \\
\hline 1 & Coalinga, 1983 & Oil fields-skunk Hollow & 10.7 & 5.09 \\
2 & Lytle Creek, 1970 & Wrightwood-6074 Park Dr & 12.4 & 5.33 \\
3 & Coyote Lake, 1979 & $\begin{array}{l}\text { Coyote lake dam (SW } \\
\text { Abut) }\end{array}$ & 6.1 & 5.74 \\
4 & Friuli, Italy, 1976 & San Rocco & 14.5 & 5.91 \\
\hline
\end{tabular}

By Considering the pressure of urban gas pipeline which is about 4 bars, through holes with diameter of 25 to $40 \mathrm{~mm}$, gas leaks in 5 weights of $0.27,0.54,0.63,0.81$ and $0.99 \mathrm{~kg}$ which are equal to 3,5, 7, 9 and $11 \mathrm{~kg}$ of TNT respectively. Diameter of hole in damaged pipe and leaking gas weight are determined in order that the building experiences the least and the most failures realistically.

\subsection{Simulation of considered scenarios}

In first step, for each of the columns hit by the explosion at the distance $R$ from the center of the charge, given the amount of explosive $w$, the reduced distance $Z=R / \sqrt[3]{w}$ is calculated. Then a triangular impulse loading is considered to be acting on the columns (see Fig. 10), whose parameters $\mathrm{P}_{0}$ (maximum initial pressure) and $t$ (duration of the

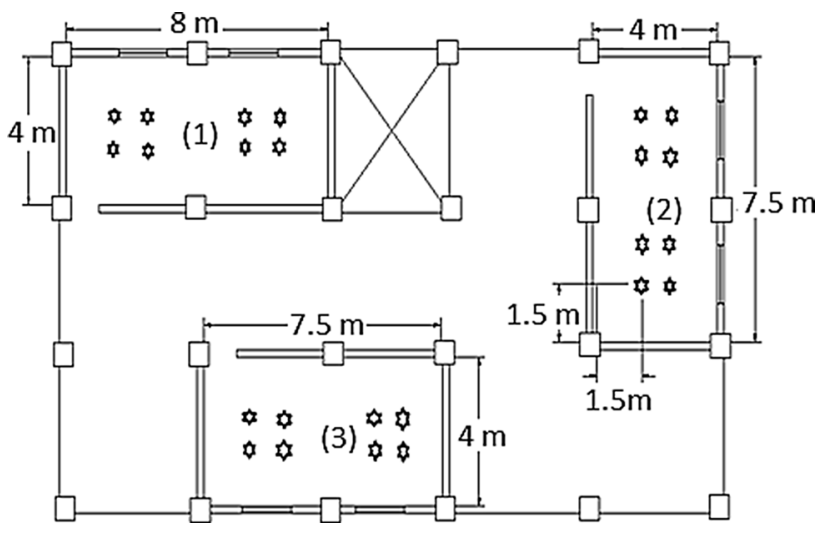

Fig. 18 Probable points of gas-explosion scenario event

impulse) were illustrated in pervious section. It is further assumed that the intensity of the impact loading is uniform across the column height. Furthermore, since such load generally acts in a direction that is not parallel to local axes of the column, it is divided into two components and both of them act to the column simultaneously and used to verify whether the column fails.

Simulation of the blast effect is done by ABAQUS software. The columns are modeled by the S4R shell element; both ends of the columns were fixed in all degrees. The column was meshed sweep with hex-dominated elements. Moreover, the blast load was applied only on one face of the column which was straightly affected by blast. Furthermore, this load was divided into two components in $\mathrm{x}$ and $\mathrm{y}$ directions depend on the angle between gas explosion position and the column and were calculated for each column.

In the interest of reducing computational time, it is important to use the smallest number of finite elements for each column member without affecting the accuracy.

With regard to limitation in experimental studies in explosion field, for validating the modeling in ABAQUS software, first a plate under blast loading according to Maleki and Rahmaneieyan (2011) was modeled and results were compared. The results were similar and hence the 
modeling was fine. Therefore, all samples were modeled in the same way.

Next step is to use a simulation technique to generate all scenario realizations, assuming that the structure is subjected to $1.2 \mathrm{DL}+0.5 \mathrm{LL}$ gravity loads and as well as the $0.002 \Sigma \mathrm{P}$ lateral loads. Also all the columns that failed in blast scenario were removed and plastic hinges assigned to the rest of columns in two positions (start and end of columns) and all the beams in three positions (start, middle and end of beams). SAP [25] provides default hinge properties and recommends P-M2-M3 hinges for columns and $M 3$ hinges for beams. Default hinges assigned to the elements (P-M2-M3 for columns and M3 for beams). Exceeding CP performance point in plastic hinges of the beams and LS performance point in those of the columns indicate the incidence of the progressive collapse in the building.

\subsection{Reliability and risk assessment of the building}

After progressive collapse analysis for each probable scenario of gas-pipe explosion in three positions for power house each with $33.3 \%$ probability following each of 4 earthquakes with probability of $25 \%$, probability of failure is determined by Eq. (6). Analysis results show that out of 480, 192 scenarios caused progressive collapse in the building. Within these 192 scenarios, 96 cases are caused by explosion of $810 \mathrm{~g}$ methane gas and 96 other cases are caused by $990 \mathrm{~g}$ of the gas. Therefore this study shows that the building is not safe against these weights of explosive material and needs more strength and reinforcement. Explosion caused by $270 \mathrm{~g}$ of methane, equal to $3 \mathrm{~kg}$ TNT, does not damage building columns showing safety of building against this explosive weight. Under the explosions caused by $540 \mathrm{~g}$ and $630 \mathrm{~g}$ of methane, equal to 5 and $7 \mathrm{~kg}$ of TNT respectively, the column near the explosion point is damaged, but the building saves its stability and progressive collapse does not occurred by omitting one failed column. Finally, by conditional theorem of Eq. (6), probability of progressive collapse in each of the installation positions of assumed power house is 13.3\% and the whole building's progressive collapse probability is $40 \%$. Therefore, regarding Eq. (7), the reliability of building is approximately $60 \%$.

\section{Conclusions}

In seismic-resistant design of structures, it is expected that no structural damage occurs in buildings and the buildings reside in operational state under moderate earthquakes. However after such earthquakes, occurrence of structural damage and loss of building operationally is still probable.
Among the most important risks is the explosion in the gas pipes of power plants after the earthquake which occurs due to the mechanical damage sustained by these pipes and their connections. In this research, a method for estimating probability of the progressive collapse and the reliability of the steel building while encountering medium earthquakes followed by explosion in the gas pipeline was presented. According to the assumptions and analyses in the numerical example, these results were obtained:

- By probabilistic relations of the risk assessment and under the given assumptions, occurrence probability of progressive collapse in the building is $40 \%$ and reliability is determined as $60 \%$.

- By simulating the probable points of gas-explosion scenarios following medium earthquakes and their effect on the building, the safety and the vulnerability of the building can be assessed in a logic approach to detect vulnerable elements of the structure.

- It became clear that the studied building is completely safe under gas explosion limited to $270 \mathrm{~g}$ (equal to $3 \mathrm{~kg}$ TNT) and even no local damage is observed.

- The building experiences local damage due to gas explosion with weight up to $630 \mathrm{~g}$ (equivalent to the weights of 5 to $7 \mathrm{~kg}$ TNT) but it retains its integrity and stability so that progressive collapse is prohibited after sudden loss of some structural elements.

- However, the building is very fragile against gas explosion with weight more than $810 \mathrm{~g}$ (equal to $9 \mathrm{~kg}$ of TNT) causing local damage and consecutive progressive collapse. Hence, the building needs strengthening in such cases.

- Finally, it is worthwhile to note that under medium earthquakes, even by dropping off the building gas flow, the amount of remaining gas in the buildings gas pipes may be more than ones applied in this research, and can highly increase damages. This fact shows the importance of this research to design a flexible gas system and to increase the building resistance.

\section{References}

1. Abdollahzadeh GR, Faghihmaleki H (2018) Proposing a method for robustness index evaluation of the structures based on the risk analysis of main shock and aftershock. Int J Steel Struct. https://doi.org/10.1007/s13296-018-0081-3

2. Abdollahzadeh GR, Faghihmaleki H (2018) Proposal of a probabilistic assessment of structural collapse concomitantly subject to earthquake and gas explosion. Front Struct Civ Eng 12(3):425-437

3. Abdollahzadeh GR, Faghihmaleki H (2017) Probabilistic twohazard risk assessment of near-fault and far-fault earthquakes 
in a structure subjected to earthquake-induced gas explosion. J Build Eng 13:294-304

4. ANSI/AISC 360-05 (2005) Specification for structural steel buildings. An American National Standard, New York

5. Asprone D, Jalayer F, Prota A, Manfredi G (2010) Proposal of a probabilistic model for multi-hazard risk assessment of structures in seismic zones subjected to blast for the limit state of collapse. Int J Struct Saf 32:25-34

6. Bangash MYH, Bangash T (2006) Explosion-resistant buildings. Springer, Berlin

7. CCPS (Center for Chemical Process Safety) (1999) Guideline for consequence analysis of chemical release. American Institute of Chemical Engineers (AICHE), New York

8. Cornell EP (1994) Quantitative safety goals for risk management of industrial facilities. Struct Saf 13(3):145-157

9. Cranz C (1926) lehrbuch Der Ballistic. Springer, Berlin

10. FEMA-356 (2000) Standard and commentary for the seismic rehabilitation of buildings. The American Society of Civil Engineers for the Federal Emergency Management Agency, Washington, DC

11. Faghihmaleki $\mathrm{H}$, Najafi EK, Aini AH (2017) Seismic rehabilitation effect in a steel moment frame subjected to tow critical loads. Int J Struct Integr 8(1):1-11

12. Faghihmaleki H, Nejati F, Roshan AM, Motlagh YB (2017) An evaluation of multi-hazard risk subjected to blast and earthquake loads in RC moment frame with shear wall. J Eng Sci Technol 12(3):636-647

13. Fu F (2013) Dynamic response and robustness of tall buildings under blast loading. Constr Steel Res 80:299-307

14. Fu H, Zhang J, Jiang J, Wang Z (2017) A ductility-centred analytical model for axially restrained double-span steel beam systems subjected to sudden columns loss. Structures 10:197-208

15. Heng P, Hjiaj M, Battini JM, Limam A (2017) An enhanced SDOF model to predict the behaviour of a steel column impacted by a rigid body. Eng Struct 152:771-789

16. Hopkinson B (1915) British ordnance board minutes, 13565. The National Archives, Kew

17. Hassanifard S, Zehsaz M, Esmaeili F (2011) Spot weld arrangement effects on the fatigue behavior of multi-spot welded joints. J Mech Sci Technol 25(3):647-653

18. Jamnani $H H$, Abdollahzadeh GR, Faghihmaleki H (2017) Seismic fragility analysis of improved RC frames using different types of bracing. J Eng Sci Technol 12(4):913-934
19. Lendecker EV, Ellingwood BR (1976) Design methods for reducing the risk of progressive collapse in buildings. Building Science Series No. 98. National Bureau of Standards, Washington, DC

20. Naji A (2019) Progressive collapse analysis of steel moment frames: an energy-based method and explicit expressions for capacity curves. J Perform Constr Facil 33(2):04019008

21. Ngo T, Mendis P, Upta A, Ramsay J (2007) Blast loading and blast effects on structures-an overview. EJSE Spec Issue Load Struct 7:76-91

22. Nowak AS, Collins KR (2000) Reliability of structures. McGraw Hill, New York

23. Parisi F, Augenti N (2012) Influence of seismic design criteria on blast resistance of RC framed buildings: a case study. Eng Struct 44:78-93

24. Research of Iran Oil Industry (2002) Analysis unit of physical, chemical and Laboratory standard of gases and petroleum liquids. Research of Iran Oil Industry, Tehran

25. SAP 2000 (2015) Structural analysis program (SAP) software, computer and structure company (CSI). Version 18.0.1

26. Shiping $H$ (1993) Seismic design of buildings in China. Earthq Spectra 9(4):703-737

27. Standard 2800 (2013) Iranian code of practice for seismic resistant design of buildings, 4th edn. Building and Housing Research Center, Tehran

28. Stewart MG, Netherton MD, Rosowsky DV (2006) Terrorism risks and blast damage to built infrastructure. Nat Hazards Rev (ASCE) 7(3): 114

29. TM5-1300 (1990) Structures to resist the effects of accidental explosions. Department of the Army

30. Uang CM (1993) An evaluation of two-Level seismic design procedure. Earthq Spectra 9(1):121-135

31. UFC-4-023-03-Change 3 (Unified Facilities Criteria) (2016) Design of buildings to resist progressive collapse. Department of Defense United States of American, Arlington

Publisher's Note Springer Nature remains neutral with regard to jurisdictional claims in published maps and institutional affiliations. 